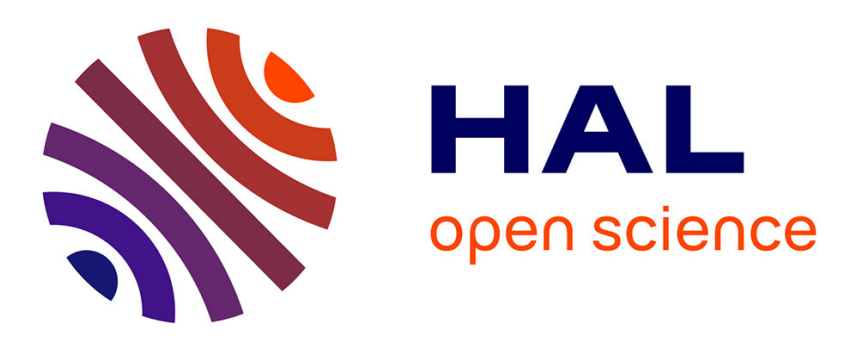

\title{
Viscosimètre à corps chutant permettant de procéder à des mesures de viscosité de liquides sous hautes pressions
}

D. Ducoulombier, F. Lazarre, H. Saint-Guirons, P. Xans

\section{- To cite this version:}

D. Ducoulombier, F. Lazarre, H. Saint-Guirons, P. Xans. Viscosimètre à corps chutant permettant de procéder à des mesures de viscosité de liquides sous hautes pressions. Revue de Physique Appliquée, 1985, 20 (10), pp.735-740. 10.1051/rphysap:019850020010073500 . jpa-00245388

\section{HAL Id: jpa-00245388 https://hal.science/jpa-00245388}

Submitted on 1 Jan 1985

HAL is a multi-disciplinary open access archive for the deposit and dissemination of scientific research documents, whether they are published or not. The documents may come from teaching and research institutions in France or abroad, or from public or private research centers.
L'archive ouverte pluridisciplinaire HAL, est destinée au dépôt et à la diffusion de documents scientifiques de niveau recherche, publiés ou non, émanant des établissements d'enseignement et de recherche français ou étrangers, des laboratoires publics ou privés. 


\title{
Viscosimètre à corps chutant permettant de procéder à des mesures de viscosité de liquides sous hautes pressions
}

\author{
D. Ducoulombier, F. Lazarre (*), H. Saint-Guirons et P. Xans
}

Laboratoire de Physique des Matériaux Industriels, Institut Universitaire de Recherche Scientifique, Avenue Louis-Sallenave, 64000 Pau, France

(*) Société Nationale Elf-Aquitaine (Production), Avenue du Président P. Angot, 64000 Pau, France

(Reçu le 13 mars 1985, accepté le 12 juillet 1985)

\begin{abstract}
Résumé. - L'appareil décrit est du type viscosimètre " à corps chutant guidé » et est destiné à l'étude des liquides sous pression. Les mesures peuvent être effectuées jusqu'à 4000 bars dans l'intervalle de températures $(-10$, $+200^{\circ} \mathrm{C}$ ). Cette publication a pour objet de présenter les différentes parties de cet appareillage, ses caractéristiques et d'analyser ses performances.
\end{abstract}

Abstract. - The instrument described is a " guided falling body " viscosimeter used for high pressure viscosity measurements. Data may be obtained up to 4000 bars in the temperature range -10 to $+200{ }^{\circ} \mathrm{C}$. This present paper gives a general description of the instrument design and capabilities.

Les méthodes de mesures de la viscosité sous hautes pressions sont en général celles utilisées à la pression atmosphérique et adaptées aux conditions de travail sous haute pression. Le lecteur pourra consulter des mises au point bibliographiques (Lazarre 1950, McCoy 1976, Persoz et Vourlat 1967, Roux et Maloubier 1959, Ducoulombier 1984).

L'appareil décrit est du type viscosimètre « à corps chutant guidé " et est destiné à l'étude des liquides sous pression. Les mesures peuvent être effectuées jusqu'à 4000 bars dans l'intervalle de températures $\left(-10,+200^{\circ} \mathrm{C}\right)$.

Le tableau synoptique (Fig. 1) présente les différentes parties de l'appareillage que nous développons ci-dessous.

\section{Cellule et tube de mesure.}

Le bloc de mesures est représenté sur la figure 2 .

L'étanchéité de la cavité interne de la cellule est assurée par un joint auto-serreur ; une huile de pression permet de maintenir l'ensemble à la pression souhaitée et un dispositif thermostatique (non représenté) maintient une température constante contrôlée par un couple thermo-électrique (fer-constantan) situé à l'intérieur de l'autoclave. Le tube de mesure calibré de diamètre $(6,70 \pm 0,01 \mathrm{~mm})$ et de longueur utile $(16,6 \pm 0,1 \mathrm{~cm})$ est entouré de deux enroulements de fil électrique destinés à détecter le passage du corps

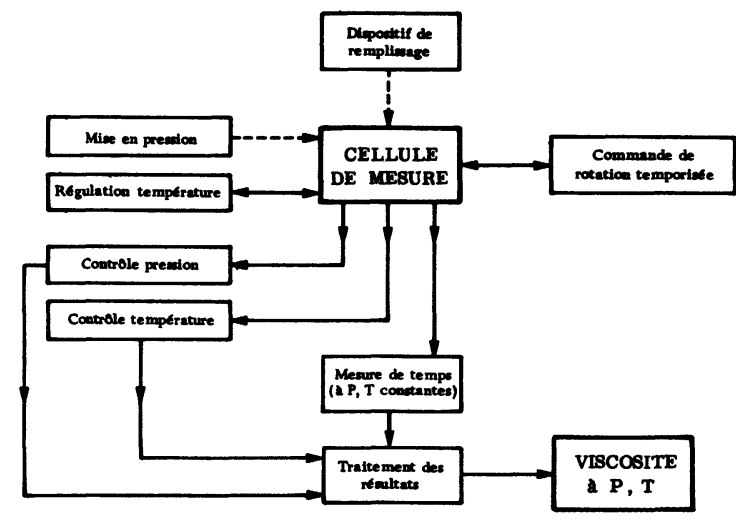

Fig. 1. - Tableau synoptique de la mesure.

[Synoptic table of measure.]

chutant. Les liaisons électriques sont assurées à travers l'enceinte par des passages isolés et étanches spéciaux obturés d'araldite polymérisée.

Un dispositif annexe comprenant un ensemble " compresseur pneumatique, pompe à vide " permet d'introduire le fluide à étudier dans le tube de mesure.

Le viscosimètre étant vérouillé verticalement le solide plongeur tombe par gravité; la mesure terminée, un système automatique temporisé assure le retournement de la cellule déclenchant un nouveau cycle 


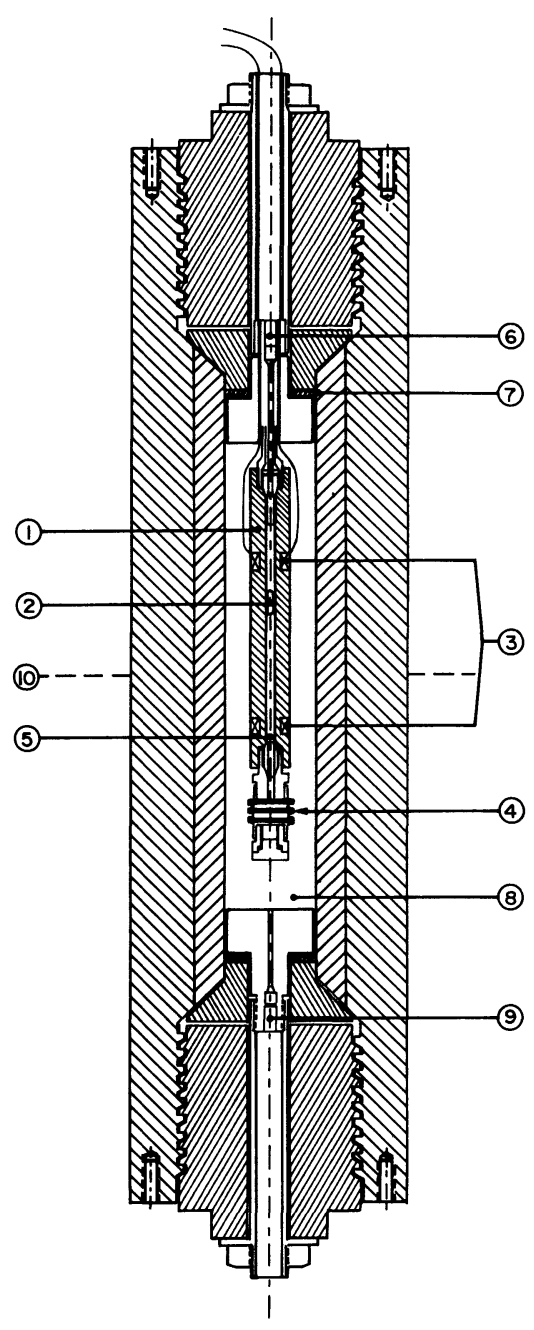

Fig. 2. - Bloc de mesure de viscosités sous pression. 1) Tube de mesure; 2) Corps chutant; 3) Bobines de détection ; 4) Soufflet (Téflon); 5) Liquide à étudier ; 6) Entrée du liquide à étudier ; 7) Joint d'étanchéité ; 8) Huile de pression; 9) Entrée du fluide compresseur (huile de pression); 10) Axe de rotation de la cellule.

[High pressure viscosity measuring vessel.

1) Tube for measure; 2) Falling body; 3) Detection coils; 4) Bellow (Teflon); 5) Studied liquid ; 6) Entrance of studied liquid; 7) Gasket; 8) High-pressure oil ; 9) Entrance of the compressor fluid (high-pressure oil); 10) Axis of rotation of the cell.]

permettant ainsi d'effectuer un nombre de mesures prédéterminé.

\section{Corps chutant.}

Dans ce type d'appareil le corps chutant est un cylindre métallique, aux extrémités hémisphériques pouvant circuler dans le tube calibré contenant le liquide à étudier; l'espace annulaire est dimensionné de façon que l'on reste en régime laminaire durant le temps de chute; le corps chutant comporte sur ses flancs des tiges guides afin d'éviter les oscillations et de permettre une chute rigoureusement axiale; nous disposons
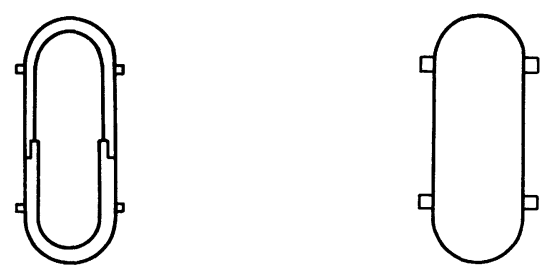

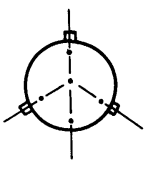

(o)

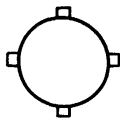

(b)
Fig. 3. - Corps chutant : a) creux; b) plein.

[Falling body : a) hollow; b) solid.]

d'une gamme de cylindres pleins ou creux (Fig. 3) plus ou moins lestés et de diamètres différents dont l'utilisation est fonction du liquide étudié et du domaine pression-température analysé.

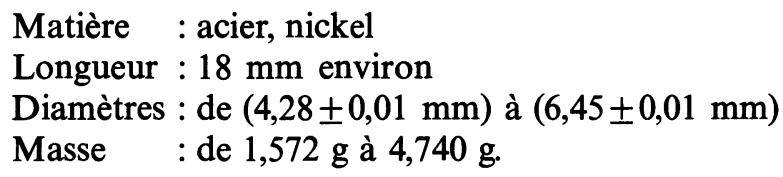

\section{Détection du passage du cylindre chutant.}

2.1 ENROULEMENTS. - Chaque ensemble de bobines comporte un primaire de $60 \Omega$ de résistance et un secondaire de $150 \Omega$, les deux enroulements secondaires sont branchés en série et en opposition de phase. Le passage des fils au travers de l'enceinte est réalisé par l'intermédiaire de cosses collées sur des céramiques coniques, elles-mêmes collées à l'araldite sur les parois internes; les fils sont vissés sur les cosses à l'intérieur de la cellule et soudés à l'extérieur.

2.2 SignAUX ÉLECTRIQUES. - Les primaires reçoivent une tension alternative de fréquence et d'amplitude réglables (en général $300 \mathrm{~Hz}, 5 \mathrm{~V}$ ).

En l'absence du corps chutant le signal induit correspondant est déterminé par la masse métallique du tube et la nature du liquide à étudier. Lorsque le cylindre traverse une bobine la masse magnétique efficace résultante devient plus importante et le signal induit dans le secondaire présente un maximum. L'allure générale d'un profil de variation est représentée sur la figure $4 ; V_{s}(t)$ représente la variation dans le temps de la tension redressée utilisée pour la détection.

2.3 DéteCTeUR De MAXIMuM. - Le système de détection correspond à un détecteur de maximum à armement à seuil (Fig. 5) (brevet déposé) (Lazarre et 


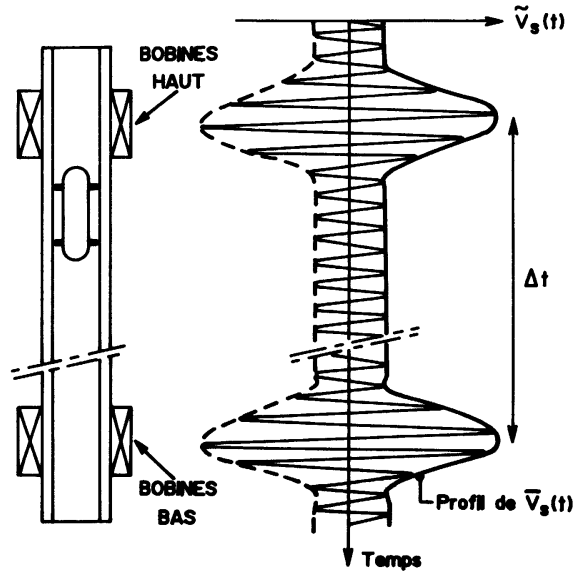

Fig. 4. - Variations de la tension de détection en fonction du parcours du corps chutant.

[Variations of the detection voltage as a function of the falling body travel.]

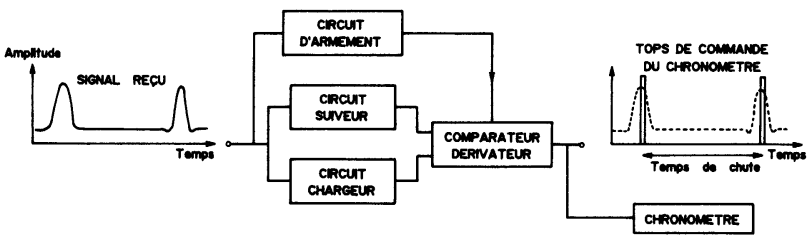

Fig. 5. - Détecteur de maximum à armement au seuil.

[Detector of maximum.]

Ducoulombier 1982). Il comporte essentiellement un amplificateur différentiel relié à un circuit suiveur et à un circuit de charge.

Cet appareil possède un circuit qui détecte une décroissance faible dans la variation lente d'une tension; ce circuit armé lorsque le signal d'entrée atteint un seuil donné délivre un top de commande lorsque la décroissance s'amorce après l'obtention du maximum, déclenchant la mise en fonctionnement ou l'arrêt du chronomètre.

Un enregistreur graphique branché en parallèle permet de contrôler si nécessaire la forme du signal et l'intervalle de temps.

\section{Mise en pression et contrôle.}

La mise en pression de l'ensemble de mesure suppose son remplissage préalable par un fluide transmetteur. Ce fluide joue un rôle important dans la stabilisation de la température et de la pression après modification de l'une ou l'autre.

La mesure des pressions peut s'effectuer suivant la gamme par deux chaînes de mesure 1000 et 4000 bars. Chaque chaîne est constituée d'un capteur à jauge métallique et d'une unité de conditionnement à sortie analogique munie d'un afficheur digital. Les préci- sions sont à $\pm 0,1$ bar pour l'échelle 1000 bars et \pm 2 bars pour l'échelle 4000 bars, les fluctuations de pression étant inférieures à 0,1 bar. Le capteur est monté sur le tubing de liaison cellule-pompe, près du raccord de la cellule.

\section{Régulation de la température et contrôle.}

Le chauffage de la cellule est assuré par un collier chauffant monté au niveau de l'axe de rotation (Fig. 6). Une sonde platine $100 \Omega$ montée contre la cellule est reliée à un thermostat de précision préalablement étalonné. Celui-ci régule la température à $\pm 0,1^{\circ} \mathrm{C}$ par contrôle proportionnel du courant envoyé au collier chauffant.

Un dispositif de refroidissement complémentaire permet de descendre en dessous de la température ambiante. Deux ensembles de conduits thermiques semi-cylindriques entourent la cellule à ses extrémités et sont branchés en parallèle sur un échangeur. Suivant le fluide réfrigérant utilisé cet ensemble autorise la couverture du domaine de températures $-10^{\circ} \mathrm{C}$, $+200^{\circ} \mathrm{C}$.

Le contrôle est effectué par un thermocouple ferconstantan relié à un afficheur digital à sortie analogique qui couvre largement le domaine concerné à $\pm 1^{\circ} \mathrm{C}$

Le thermocouple est installé à l'intérieur de l'enceinte pressurisée et baigne dans l'huile de pression près du soufflet.

\section{Dispositif de remplissage/dégazage.}

Un dispositif complexe « Pompe à vide, vérin pneumatique, résistance chauffante " (Fig. 7) permet, en fonction de la fluidité du liquide, d'effectuer cette opération selon différentes procédures; on peut ainsi introduire des produits dont la température de fusion est voisine de $+60^{\circ} \mathrm{C}$.

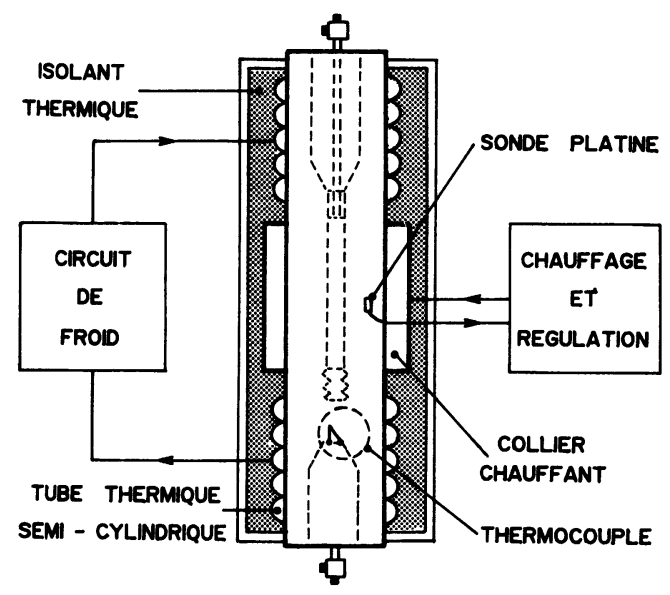

Fig. 6. - Régulation et contrôle de température.

[Schematic of temperature control.] 


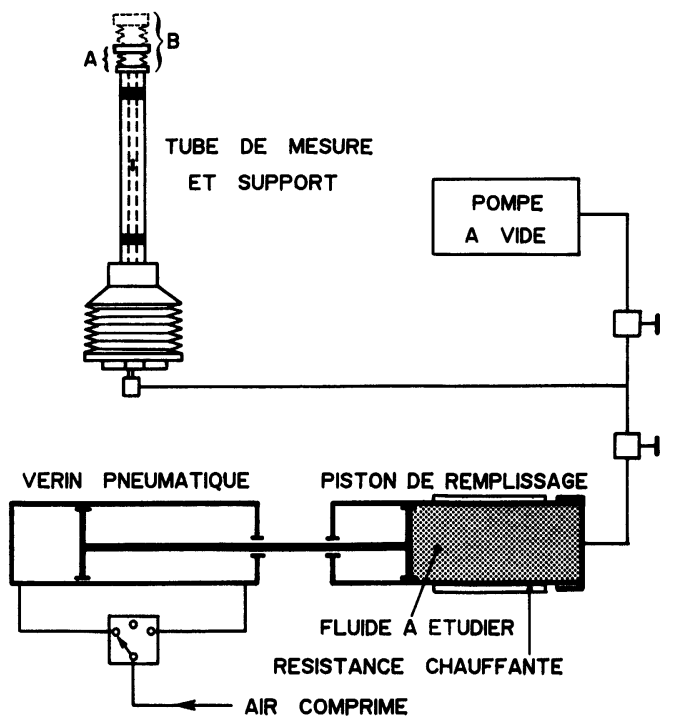

Fig. 7. - Procédure de remplissage du tube de mesure.

[Filling procedure of the sample tank.]

\section{Acquisition des données et commande du viscosi- mètre.}

Un ordinateur Apple II + , 64 K-octets, muni de deux lecteurs de disquettes et d'une imprimante a pour rôle, d'une part, la saisie des données et d'autre part la commande de rotation de la cellule grâce à l'adjonction de cartes d'acquisition et de commande. Pour entrer plusieurs paramètres et réaliser l'adaptation adéquate des signaux électriques, un circuit de multiplexage et de conditionnement des tensions complète le bloc informatique.

Sur la figure 8 sont explicitées les fonctions de chacune de ces cartes.

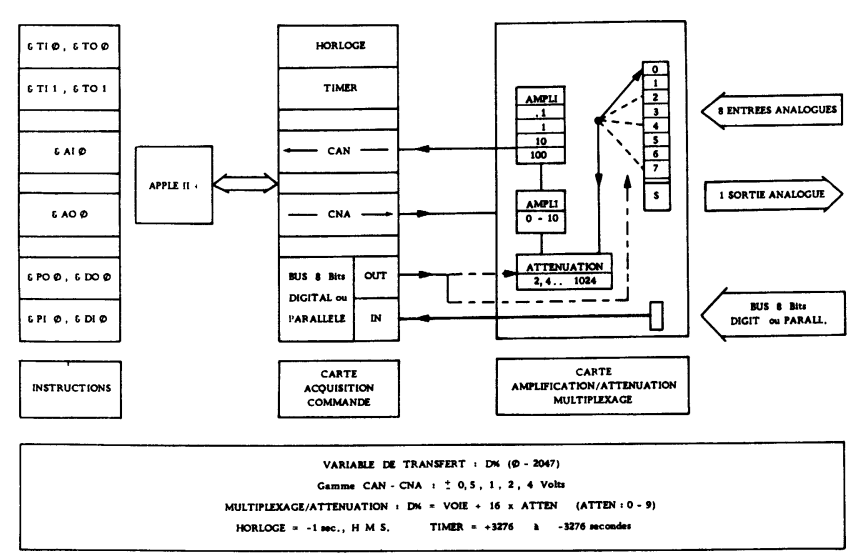

Fig. 8. - Acquisition de données.

[Schematic of data acquisition system.]

\section{Mesures.}

La valeur du coefficient de viscosité d'un fluide à $P$ et $T$ est donnée pour un appareillage du type « corps chutant " par la relation :

$$
\underset{\mathrm{cP}}{\eta_{P, T}=K\left(\rho_{\mathrm{sol}} \underset{\mathrm{g}}{\downarrow} \rho_{\mathrm{liq}}\right)} \underset{\mathrm{g} / \mathrm{cm}^{3}}{\Delta} \stackrel{\mathrm{s}}{\downarrow}
$$

$\Delta t \quad$ le temps de chute

$\rho_{\text {liq }}$ la masse volumique du liquide étudié

$\rho_{\text {sol }}$ la masse volumique du corps chutant

$K$ est une fonction de l'appareillage utilisé

- La détermination de la masse volumique de chacun des " corps chutants " utilisés est effectuée à l'aide d'un picnomètre de précision.

- La détermination de la masse volumique des fluides étudiés repose à la fois sur les valeurs trouvées dans la littérature et les mesures réalisées. On tient compte des variations de ce paramètre pour un liquide donné en fonction de la température et de la pression.

- Le temps de chute $\Delta t$, fonction des fluctuations de vitesse du corps chutant et de la sensibilité du dispositif de détection, nécessite d'effectuer plusieurs mesures dont les écarts à la moyenne sont inférieurs à $1 \%$.

7.1 Valeurs de $K$ et variations. - A pression atmosphérique, pour les gammes de viscosités et de températures couvertes, le coefficient $K$ (pour un corps chutant déterminé) n'apparaît pas fonction de la température; en ce qui concerne l'éventuelle variation de ce coefficient avec la pression, se pose le problème du caractère "étalon " des références viscosimétriques. Considérant $K$ indépendant de la pression, la comparaison des valeurs de la viscosité obtenues pour l'hexadécane (Fig. 9) avec celles données par l'ASME (présentées comme valeurs absolues) montre une cohérence d'ensemble tout à fait satisfaisante qui nous incite à penser que dans le domaine pressiontempérature étudié nous pouvons supposer le coefficient $K$ indépendant de la pression.

Rappelons que dans ce type de viscosimètre on force le fluide à s'écouler dans un espace annulaire très petit et que dans ces conditions la résistance à l'avancement est due à trois causes :

- la déformation des lignes d'écoulement avant et après l'obstacle, que l'on minimise en employant des corps chutants longs;

- au gradient de pression correspondant à l'écoulement du fluide dans l'espace annulaire;

- au déplacement relatif du plongeur et du tube.

En utilisant des espaces annulaires convenables pour rester en régime laminaire, le temps de chute entre deux repères pour une pression donnée est en définitive proportionnel à $\eta$ et la formule précédente applicable. 


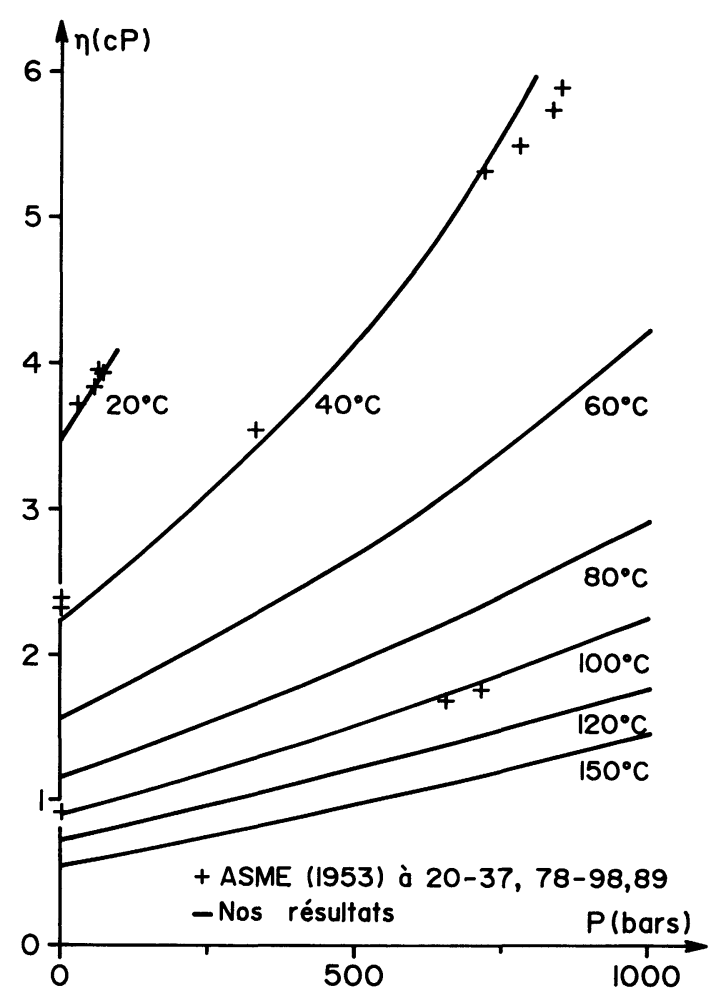

Fig. 9. - Variations de la viscosité isotherme du n-hexadécane en fonction de la pression.

[Isothermal viscosity of $\mathrm{n}$-hexadecane versus pressure.]

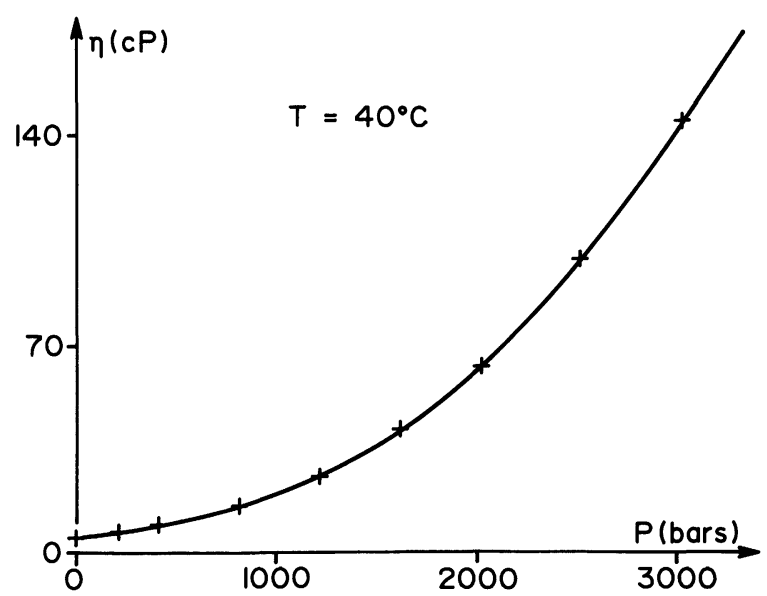

Fig. 10. - Variations de la viscosité isotherme $\left(40^{\circ} \mathrm{C}\right)$ d'une huile minérale $\mathrm{A}$ en fonction de la pression.

[Isothermal viscosity (at $40^{\circ} \mathrm{C}$ ) of a mineral oil A versus pressure.]

\section{Exemples d'applications.}

Au moyen du viscosimètre décrit ci-dessus de nombreux produits ont été étudiés, en particulier : des n-alcanes, des alkyl-benzènes, des mélanges binaires et quaternaires (publications en préparation) et des huiles minérales dont nous rapportons quelques exemples illustrant la mesure de viscosités élevées dans le domaine de pression concerné (Figs 10, 11 et 12).

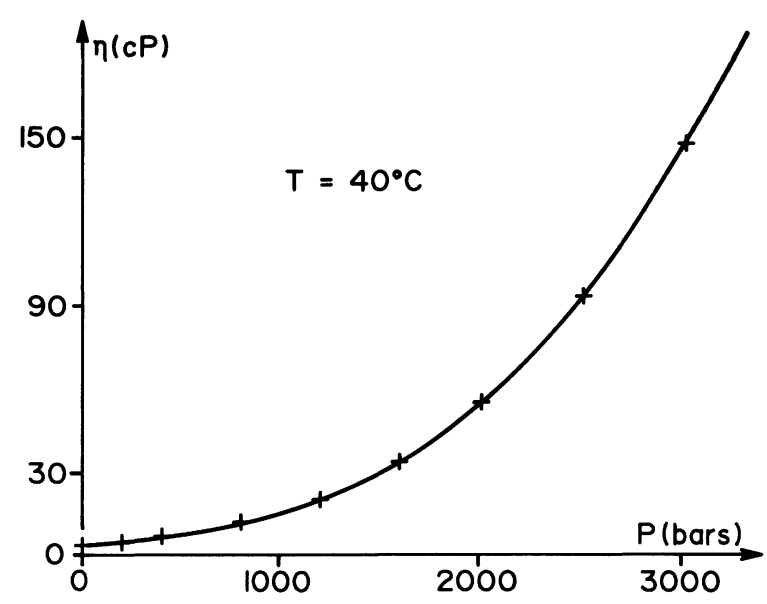

Fig. 11. - Variations de la viscosité isotherme $\left(40^{\circ} \mathrm{C}\right)$ d'une huile minérale $B$ en fonction de la pression.

[Isothermal viscosity (at $40^{\circ} \mathrm{C}$ ) of a mineral oil B versus pressure.]

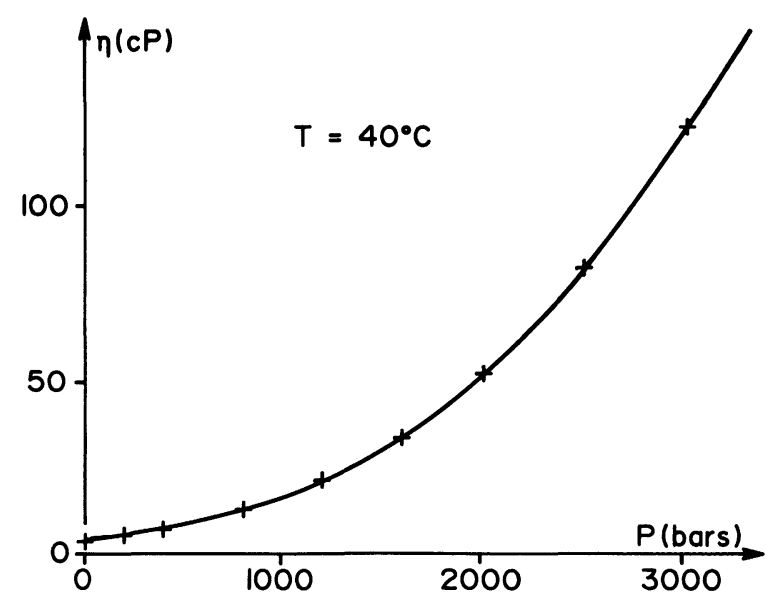

Fig. 12. - Variations de la viscosité isotherme $\left(40^{\circ} \mathrm{C}\right)$ d'une huile minérale $\mathrm{C}$ en fonction de la pression.

[Isothermal viscosity (at $40^{\circ} \mathrm{C}$ ) of a mineral oil C versus pressure.] 


\section{Bibliographie}

LAZARre, F., Viscosimétrie sous hautes pressions. J. Physique Radium 11 (1950) 12, 51.

LAZARre, F. et DucoulombIER, D., Dispositif de détection de changement de pente d'une tension variable (1982). Brev. Invention $\mathrm{n}^{\mathrm{o}} 82.21550 \mathrm{du} 22.12 .82$.

McCoy, D. D., Critical region behavior and effect of pressure on liquid viscosity. Thesis, Oklahoma State Univ. Stillwater, Oklahoma (1976).
Persoz, B. et Vourlat, P., Viscosité. Techn. Ing. (1967) p. 3305.

Roux, F. et Maloubier, F., Quelques exemples d'application du viscosimètre Charron, Rev. Inst. Fr. Pétrole (1959) 1211.

DuCOUlomBIER, D., Viscosité des liquides. Viscosimètre haute pression à cylindre chutant. Etude des alcanes et des alkylbenzènes. Thèse de 3 e Cycle (1984).

ASME Pressure-viscosity report. 1953. 